\title{
Evaluation of portable blood glucose meters using canine and feline pooled blood samples
}

\author{
A. Mori ${ }^{1}$, H. Oda ${ }^{1}$, E. Onozawa ${ }^{1}$, S. Shono ${ }^{1}$, T. Takahashi', \\ S. Yamashita ${ }^{2}$, H. Fujimoto ${ }^{2}$, T. Sako ${ }^{1}$ \\ ${ }^{1}$ School of Veterinary Nursing and Technology, Faculty of Veterinary Science, Nippon Veterinary \\ and Life Science University, 1-7-1 Kyonancho, Musashino, Tokyo 180-8602, Japan \\ ${ }^{2}$ Arkray, Inc. Yousuien-nai, 59 Gansuin-cho, Kamigyo-ku, Kyoto 604-8153, Japan
}

\begin{abstract}
This study evaluated the accuracy and reproducibility of a human portable blood glucose meter (PBGM) for canine and feline whole blood. Reference plasma glucose values (RPGV) were concurrently measured using glucose oxidation methods. Fifteen healthy dogs and 6 healthy cats were used for blood sampling. Blood glucose concentrations and hematocrits were adjusted using pooled blood samples for our targeted values. A positive correlation between the PBGM and RPGV was found for both dogs $(\mathrm{y}=0.877, \mathrm{x}=-24.38, \mathrm{r}=0.9982, \mathrm{n}=73)$ and cats $(\mathrm{y}=1.048, \mathrm{x}=-27.06, \mathrm{r}=0.9984$, $\mathrm{n}=69$ ). Acceptable results were obtained in error grid analysis between PBGM and RPGV in both dogs and cats; $100 \%$ of these results were within zones A and B. Following ISO recommendations, a PBGM is considered accurate if $95 \%$ of the measurements are within $\pm 15 \mathrm{mg} / \mathrm{d} l$ of the RPGV when the glucose concentration is $<100 \mathrm{mg} / \mathrm{d} l$ and within $\pm 15 \%$ when it is $\geq 100 \mathrm{mg} / \mathrm{d} l$; however, small numbers of samples were observed inside the acceptable limits for both dogs (11\%, 8 of 73 dogs) and cats $(39 \%, 27$ of 69 cats). Blood samples with high hematocrits induced lower whole blood glucose values measured by the PBGM than RPGV under hypoglycemic, normoglycemic, and hyperglycemic conditions in both dogs and cats. Therefore, this device is not clinically useful in dogs and cats. New PBGMs which automatically compensate for the hematocrit should be developed in veterinary practice.
\end{abstract}

Key words: cat, dog, hematocrit, plasma, whole blood

\section{Introduction}

Portable blood glucose meters (PBGMs) are widely used for the management of diabetes mellitus in dogs and cats. Various recently developed PBGMs are now commercially available from several companies. However, canine and feline blood glucose concentrations obtained from human PBGMs are com- monly lower than those obtained from the hexokinase or glucose oxidase reaction, which is used to determine reference glucose values. Thus, the difference between blood glucose concentrations obtained from human PBGMs and reference glucose values should be evaluated before using PBGMs for dogs and cats. The GLUCOCARD X-mini plus (Arkray, Kyoto, Japan) provides rapid and highly accurate test results

Correspondence to: A. Mori, e-mail: amori@nvlu.ac.jp 
for human blood samples. The required sample volume is quite small (approximately $0.3 \mu l$ ). The measuring time is $5 \mathrm{sec}$, and the measuring range is 10 to $600 \mathrm{mg} / \mathrm{d} l$. However, this device has not been used for dogs and cats.

To evaluate the system accuracy and measurement reproducibility of newly developed PBGMs for veterinary practice, sufficient numbers of canine and feline hypoglycemic, normoglycemic, and hyperglycemic blood samples are needed. However, large numbers of diabetic dogs and cats are required for such experiments. Furthermore, side effects are inevitable when taking blood samples from animals in a hypoglycemic condition. Based on the International Organization for Standardization (ISO) recommendations, if hypoglycemic or hyperglycemic blood samples from diabetic patients are unavailable, euglycemic whole blood (WB) samples should be incubated to allow for erythrocyte glycolysis or a glucose solution should be added (Brito-Casillas et al. 2014; ISO 15197 2003, ISO 15197 2013). Using the above-described methods, the device accuracy can be more easily and safely evaluated using healthy canine and feline blood samples. Additionally, higher hematocrit values interfere with glucose measurement when using PBGMs (Brito-Casillas et al. 2014, Domori et al. 2014). Therefore, it is also necessary to evaluate whether changes in hematocrit values affect glucose values measured by the GLUCOCARD X-mini plus.

The objective of this study was to compare the system accuracy and measurement reproducibility in canine WB measured by a PBGM device with reference glucose values. Pooled blood samples were used to achieve this objective. This is an easy and useful method for adjusting glucose concentrations and hematocrit values without using diabetic or hypoglycemic dogs and cats (ISO 15197 2003, ISO 15197 2013).

\section{Materials and Methods}

In total, 15 healthy dogs ( 8 castrated male and 7 spayed female beagles, 2-11 years old, $8.0-14.0 \mathrm{~kg}$ ) and 6 healthy cats ( 6 castrated male domestic shorthairs, 2-8 years old, $4.9-6.0 \mathrm{~kg}$ ) were used for this study. All dogs and cats were kept and maintained at the Nippon Veterinary and Life Science University. All dogs and cats were tested for concurrent diseases by physical examination, complete blood cell counts, and serum biochemistry tests. This study was approved by the Nippon Veterinary and Life Science University Animal Research Committee.

Preprandial blood (6 $\mathrm{m} l$ for dogs, $10 \mathrm{ml}$ for cats) was withdrawn from the jugular vein of all 15 dogs and 6 cats and placed into heparinized plastic tubes. WB and plasma samples from 73 samples (from 15 dogs) and 69 samples (from 6 cats) were used to assess accuracy. The collected venous blood samples were dispensed into tubes of the determined numbers (73 samples for dogs, 69samples for cats). Some dispensed tubes were used for normoglycemic blood samples. A highly concentrated glucose solution was added to some dispensed tubes to prepare samples at each concentration and create hyperglycemic blood samples. The volume of glucose solution was $\leq 1 \%$. To reduce the glucose concentration, residual tubes were stored in a $36^{\circ} \mathrm{C}$ water bath for 1 to $8 \mathrm{hr}$ to allow for erythrocyte glycolysis and thus prepare the various hypoglycemic blood samples.

Normoglycemic and adjusted blood samples were measured with a GLUCOCARD X-mini plus PBGM. This device was operated and calibrated according to the manufacturer's instructions. Residual WB samples were immediately centrifuged at $2000 \times g$ (PMC-060 Capsulefuge; Tomy, Tokyo, Japan) for $10 \mathrm{~min}$ to obtain the plasma and red blood cell fractions. The Glucose concentrations of each plasma sample were measured by the glucose oxidation method using an ADAMS Glucose GA-1153 analyzer (Arkray) to obtain the reference glucose values. The hematocrit values were adjusted by reducing the plasma or adding plasma to the samples using pooled euglycemic samples. After adjusting the hematocrit values, the glucose concentrations were adjusted by adding glucose solutions or incubating in a $36^{\circ} \mathrm{C}$ water bath as previously mentioned. Finally, the agitated WB samples were measured with the PBGM. The WB samples were then centrifuged to obtain plasma samples for measurement of the reference glucose values.

Intra-assay reproducibility of the WB glucose concentrations was estimated using 3 pooled blood samples (hypoglycemic, normoglycemic, and hyperglycemic) with 30 replicates. Comparison of the reference glucose values $(n=1)$ with those obtained by the PBGM $(\mathrm{n}=30)$ under different hematocrit percentages was also evaluated using 3 pooled canine and feline blood samples (hypoglycemic, normoglycemic, and hyperglycemic). The hematocrit percentage was classified as low $(<37 \%)$, normal $(37 \%-54 \%)$, or high $(>54 \%)$ in dogs and low $(<30 \%)$, normal $(30 \%-47 \%)$, or high $(>47 \%)$ in cats. Hematocrit was evaluated using the conductimetry method with a SPOTCHEM SG SG-1220 (Arkray). All measurements were performed at room temperature $\left(20^{\circ} \mathrm{C}-25^{\circ} \mathrm{C}\right)$. A drop of blood was placed on the parafilm, and 30 PBGM devices were then touched to the drop of blood to measure the blood glucose concentration. All procedures were completed within $1 \mathrm{~min}$. 
Table 1. Intra-assay coefficients of variation of the portable blood glucose meter in $\operatorname{dogs}(\mathrm{n}=30)$.

\begin{tabular}{cccc}
\hline & Hypoglycemic & Normoglycemic & Hyperglycemic \\
\hline Mean & 36.5 & 71.0 & 319.1 \\
SD & 1.0 & 1.8 & 10.7 \\
CV $(\%)$ & 2.8 & 2.6 & 3.3 \\
\hline
\end{tabular}

$\mathrm{SD}=$ standard deviation; $\mathrm{CV}=$ coefficient of variation.

Table 2. Intra-assay coefficients of variation of the portable blood glucose meter assay in cats $(\mathrm{n}=30)$.

\begin{tabular}{cccc}
\hline & Hypoglycemic & Normoglycemic & Hyperglycemic \\
\hline Mean & 42.4 & 102.9 & 356.3 \\
SD & 1.2 & 2.2 & 8.5 \\
CV $(\%)$ & 2.7 & 2.2 & 2.4 \\
\hline
\end{tabular}

$\mathrm{SD}=$ standard deviation; $\mathrm{CV}=$ coefficient of variation.

Table 3. Comparison of reference glucose values and portable blood glucose meter measurements under different hematocrits in dogs.

\begin{tabular}{cccccccccccc}
\hline & & \multicolumn{1}{c}{$\mathrm{n}$} & \multicolumn{3}{c}{ Hypoglycemic } & \multicolumn{3}{c}{ Normoglycemic } & \multicolumn{3}{c}{ Hyperglycemic } \\
\hline Reference glucose value & $(\mathrm{mg} / \mathrm{d} l)$ & $\mathrm{n}=1$ & & 68.9 & & & 115.8 & & 375.8 \\
\hline $\mathrm{Ht}$ & $(\%)$ & $\mathrm{n}=1$ & 36 & 47 & 57 & 33 & 45 & 54 & 36 & 46 & 55 \\
\hline \multirow{2}{*}{ PBGM } & $\mathrm{mg} / \mathrm{d} l$ & $\begin{array}{c}\text { Mean } \\
(\mathrm{n}=30) \\
\mathrm{SD}\end{array}$ & 47.3 & 36.5 & 21.7 & 95.1 & 71.0 & 49.0 & 382.6 & 319.1 & 247.4 \\
& & & 1.1 & 1.0 & 0.6 & 3.1 & 1.8 & 1.5 & 13.4 & 10.7 & 6.4 \\
\hline
\end{tabular}

$\mathrm{Ht}=$ hematocrit; PBGM = portable blood glucose meter.

Table 4. Comparison of reference glucose values and portable blood glucose meter measurements under different hematocrits in cats.

\begin{tabular}{cccccccccccc}
\hline & & $\mathrm{n}$ & \multicolumn{3}{c}{ Hypoglycemic } & \multicolumn{3}{c}{ Normoglycemic } & \multicolumn{3}{c}{ Hyperglycemic } \\
\hline Reference glucose value & $(\mathrm{mg} / \mathrm{d} l)$ & $\mathrm{n}=1$ & & 64.4 & & & 131.4 & & 371.9 \\
\hline $\mathrm{Ht}$ & $(\%)$ & $\mathrm{n}=1$ & 23 & 35 & 53 & 24 & 37 & 52 & 24 & 37 & 52 \\
\hline \multirow{2}{*}{ PBGM } & $\mathrm{mg} / \mathrm{d} l$ & $\begin{array}{c}\text { Mean }=30) \\
\mathrm{SD}\end{array}$ & 52.9 & 42.4 & 25.4 & 128.5 & 102.9 & 69.2 & 427.6 & 356.3 & 249.5 \\
& & & 2.2 & 1.2 & 1.0 & 3.6 & 2.2 & 2.0 & 8.6 & 8.5 & 6.1 \\
\hline
\end{tabular}

$\mathrm{Ht}=$ hematocrit; PBGM = portable blood glucose meter.

In the statistical analysis, values are expressed as mean \pm standard deviation (SD). Spearman's rank correlation coefficient was used to identify significant correlations between measurements, and Spearman's correlation coefficient (r) was obtained using GraphPad Prism 6 (GraphPad Software, Inc., San Diego, CA). PBGM data were evaluated using the clinical relevant error grid analysis (Clarke et al. 1987, Cox et al. 1997). The error grid compares values measured by reference glucose values (x-axis) versus the PBGM values (y-axis) in 5 zones associated with the following 5 risk levels: zone $\mathrm{A}$, clinically accurate; zone B, altered clinical action but no or minimal effect on clinical outcome; zone $\mathrm{C}$, altered clinical action with a likely effect on clinical outcome; zone D, altered clinical action with considerable medical risk; and zone E, altered clinical action with dangerous consequences
(Johnson et al. 2009). Error grid analysis was conducted using Excel 2007 SP3 (Microsoft Corporation, Redmond, WA). Bland-Altman difference plots were constructed for comparison of the results of the PBGM versus the reference glucose values using GraphPad Prism 6.

\section{Results}

When the PBGM and reference glucose values were examined for dogs and cats, a positive correlation was observed in both dogs $(\mathrm{y}=0.877, \mathrm{x}=-24.38$, $\mathrm{r}=0.9982, \mathrm{n}=73)$ and cats $(\mathrm{y}=1.048, \mathrm{x}=-27.06$, $\mathrm{r}=0.9984, \mathrm{n}=69$ ).

Because the efficacy of the GLUCOCARD $\mathrm{X}$-mini plus has not been determined in dogs and cats, 

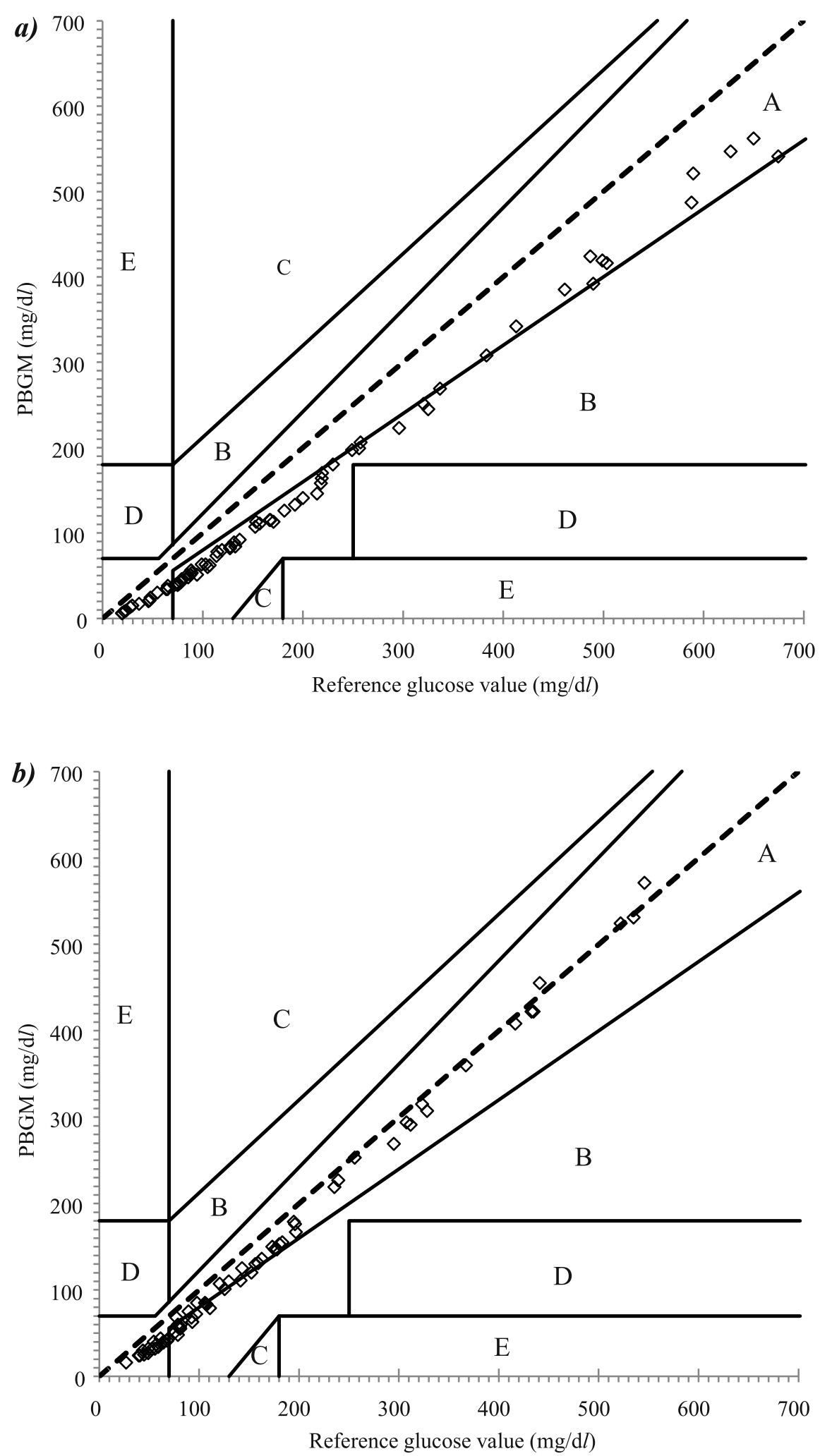

Fig. 1. Error grid analysis of blood glucose concentrations for the portable blood glucose meter (PBGM) in (a) dogs (n = 73) and $(\mathbf{b})$ cats $(\mathrm{n}=69)$. Blood glucose concentrations measured with the PBGM are plotted against reference glucose values.

it was important to assess its reproducibility and imprecision. Analytical evaluation of the glucose concentrations under hypoglycemia, normoglycemia, and hyperglycemia with the PBGM device indicated acceptable analytical imprecision, with intra-assay coefficients of variation not exceeding the commonly accepted 5\% limit (Tables 1 and 2).

Error grid analyses of all canine and feline blood 

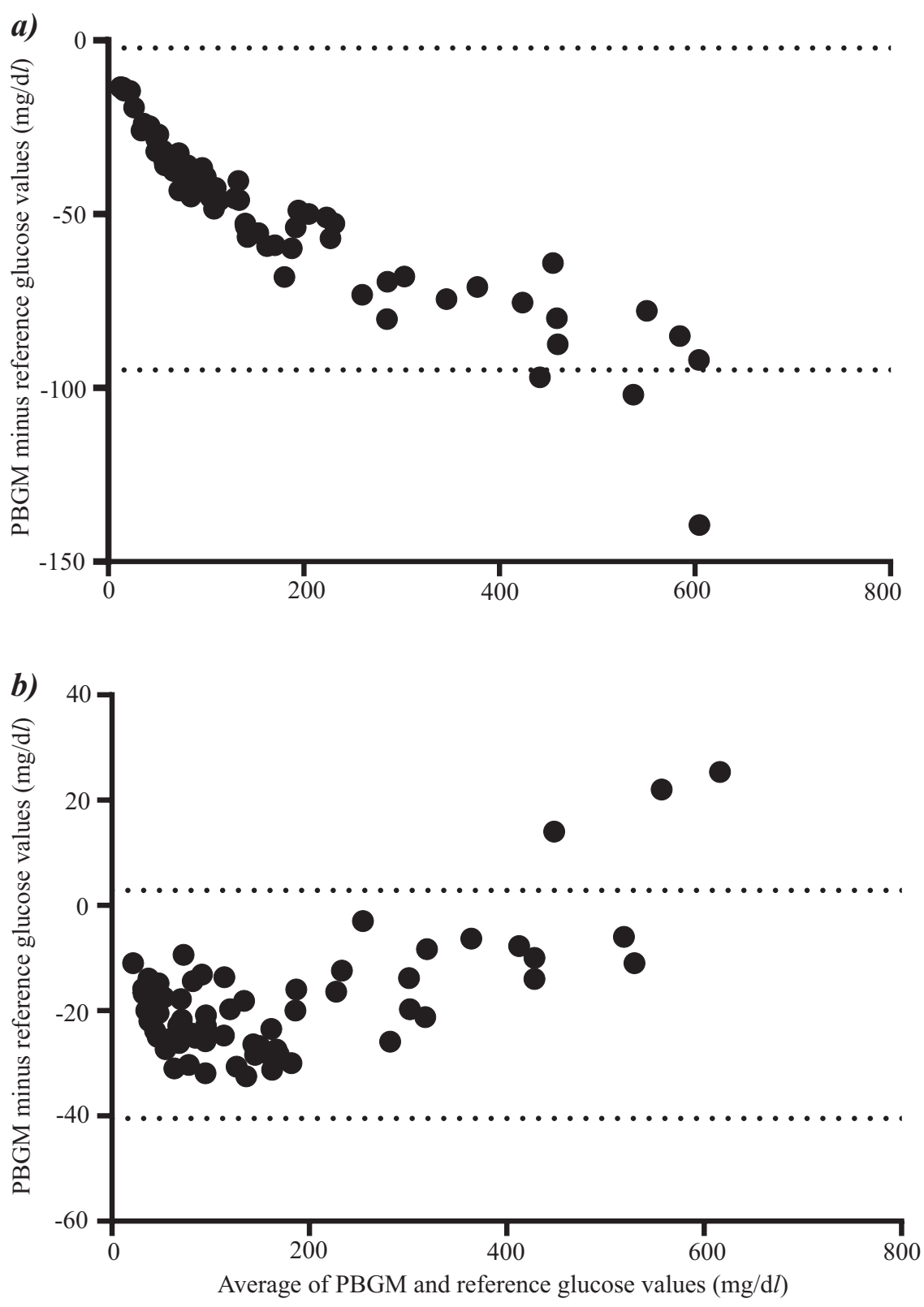

Fig. 2. Bland-Altman difference plots of blood glucose concentrations measured with the portable blood glucose meter (PBGM) and reference glucose values in $(\mathbf{a})$ dogs $(\mathrm{n}=73)$ and $(\mathbf{b})$ cats $(\mathrm{n}=69)$. Dashed lines represent $95 \%$ limits of agreement $($ dogs, from -94.81 to -2.178 ; cats, from -40.43 to 2.881 ).

glucose measurements revealed distributions within zones A and B (Fig. 1A and B, respectively). Twenty-eight of 73 canine samples (38.4\%) and 53 of 69 feline samples $(76.8 \%)$ were plotted in zone A. Forty-five of 73 canine samples (61.6\%) and 15 of 69 feline samples $(21.7 \%)$ were plotted in zone B.

Bland-Altman plot analysis revealed a mean difference of $-48.5 \pm 23.63 \mathrm{mg} / \mathrm{d} l$ (bias $\pm \mathrm{SD}$ ) in dogs (Fig. $2 \mathrm{~A}$ ) and $-18.78 \pm 11.05 \mathrm{mg} / \mathrm{d} l$ (bias $\pm \mathrm{SD}$ ) in cats (Fig. 2B) between the PBGM and reference glucose values, respectively. For both dogs and cats, almost all values were inside the calculated $95 \%$ limits of agreement
(70 of 73 dogs, $96 \%$; 66 of 69 cats, $96 \%$ ) between the PBGM and the reference glucose values.

According to the ISO 15197:2013 requirements for human use, a PBGM is considered accurate if $95 \%$ of the measurements are within $\pm 15 \mathrm{mg} / \mathrm{d} l$ of the reference value when the glucose concentration is $<100$ $\mathrm{mg} / \mathrm{dl}$, and within $\pm 15 \%$ when the glucose concentration is $\geq 100 \mathrm{mg} / \mathrm{d} l$ (Melihn and Wagner 2014). From the above criteria, the small samples in the present study were inside the calculated acceptable limits (8 of 73 dogs, $11 \%$; 27 of 69 cats, 39\%) between the PBGM and the reference glucose values. 
Different hematocrit values were evaluated under hypoglycemic, normoglycemic, and hyperglycemic conditions in dogs and cats. The canine reference plasma glucose concentrations for hypoglycemia, normoglycemia, and hyperglycemia were 68.9, 115.8, and $375.8 \mathrm{mg} / \mathrm{d} l$, respectively. However, blood samples with high hematocrits induced lower blood glucose values than those with low hematocrits using the PBGM under hypoglycemic, normoglycemic, and hyperglycemic conditions in dogs (Table 3 ). The feline reference plasma glucose concentrations for hypoglycemia, normoglycemia, and hyperglycemia were 64.4, 131.4, and $371.9 \mathrm{mg} / \mathrm{d} l$, respectively. However, blood samples with high hematocrits induced lower blood glucose values than those with low hematocrits using the PBGM under hypoglycemic, normoglycemic, and hyperglycemic conditions in cats (Table 4).

\section{Discussion}

PBGMs can allow for rapid blood glucose monitoring with a small volume of blood in diabetic dogs and cats. However, because the accuracy and reproduction of PBGMs depend on each device, newly developed human PBGMs should be evaluated for veterinary use in dogs and cats.

A high level of correlation between the PBGM and reference glucose values was observed in dogs and cats in the present study. Furthermore, the intra-assay coefficients of variation of the PBGM were $<5 \%$ in both dogs and cats. Thus, the PBGM used in the current study showed high correlation, reliability, and reproducibility.

For clinical use of a PBGM, 99\% of the values should be within zones $\mathrm{A}$ and $\mathrm{B}$ in the error grid analysis. In our study, $100 \%$ of the data points were within zones $\mathrm{A}$ and $\mathrm{B}$ of the error grid, indicating no effect or a minimal effect on clinical outcomes. However, a low percentage $(38.4 \%)$ of canine samples were plotted in zone A. Meanwhile, a relatively high percentage $(77.9 \%)$ of feline samples were plotted in zone A. However, these percentages are lower than the results of previous studies (dogs, 99.1\%; cats, 92.5\% plotted in zone A) (Domori et al. 2014). Furthermore, according to the ISO 15197:2013 requirements, a small number of samples were inside the calculated acceptable limits (11\% of dogs, $39 \%$ of cats) between the PBGM and the reference glucose values. With respect to the Bland-Altman plots, most of the canine data (96\% of dogs, $96 \%$ of cats) were included in the $95 \%$ limits of agreement calculated from the PBGM and the reference glucose values. However, the ISO 15197:2013 requirements are considered strict clinical indices according to previous studies (Freckmann et al. 2012, Brito-Casillas et al. 2014, Khositseth et al. 2015). Therefore, the GLUCOCARD X-mini plus is not applicable for veterinary practice, especially in dogs and cats.

The current study also evaluated whether hematocrit affects the PBGM accuracy. Specific PBGMs are affected by hematocrit values; anemic dogs and cats show higher WB glucose concentrations as measured by PBGMs than plasma reference glucose concentrations (Wess and Reusch 2000a,b, Mori et al 2011, Brito-Casillas et al. 2014). As a result, the hematocrit clearly interfered with the results obtained by the PBGM used in the current study. High hematocrits induced lower WB glucose concentrations as measured by the PBGM than did low hematocrits and plasma glucose concentrations measured with the reference glucose values. We were unable to determine why the hematocrit values affected the results. Factors such as differences in red blood cell size and thickness in dogs and cats and the lower glucose permeability in the red blood cells of these animals than of humans might have contributed; however, further studies are required (Magnani et al. 1978, Higgins et al. 1982, Rendell et al. 1985, Barman et al. 1993, Mori et al. 2011).

This study has a number of limitations. First, we used pooled blood samples to evaluate the PBGM because of the small number of healthy dogs $(n=15)$ and healthy cats $(n=6)$. However, this limitation is also a strength because we were able to reduce the numbers of dogs and cats and avoid the side effects associated with hypoglycemic or hyperglycemic conditions in diabetic dogs and cats. Incubation of the samples or addition of glucose to the samples was thus performed to produce the various glucose concentrations. Second, to produce samples with various hematocrits, plasma and red blood cell RBC fractions were artificially adjusted. The samples used in the current study therefore might not be identical matches to blood samples in vivo. Third, PBGMs are typically used for capillary blood in the human clinical setting. The glucose level in capillary blood is approximately $20 \%$ to $25 \%$ higher than the glucose level in venous blood (Sacks 2006). Therefore, it is also necessary to determine the accuracy when using capillary blood samples in veterinary practice.

In conclusion, the GLUCOCARD X-mini plus is not in agreement with the reference glucose values in dogs and cats. Furthermore, changes in the hematocrit obviously affect the WB glucose concentration as measured by the GLUCOCARD X-mini plus. Therefore, this device is not clinically useful in dogs and cats. It is also important to understand the possibility that the hematocrit will affect the measurement re- 
sults of the blood glucose concentration when using PBGMs other than the GLUCOCARD X-mini plus; these PGBMs do not have a hematocrit correction function. New PBGMs which automatically compensate for the hematocrit should be developed in veterinary practice in the future. Furthermore, our analysis methods using canine and feline pooled blood sampling for evaluation of a PBGM are useful for basic data acquisition before using actual clinically diabetic animals. This is because the required numbers of dogs and cats were relatively small and we could artificially adjust their blood glucose concentration and hematocrit values without any adverse effects on the dogs and cats. In the future, if newly developed PBGMs pass the methods used in the current study, their accuracy and reproducibility may then be evaluated in clinically diabetic animals.

\section{References}

Barman BN, Ashwood ER, Giddings JC (1993) Separation and size distribution of red blood cells of diverse size, shape, and origin by flow/hyperlayer field-flow fractionation. Anal Biochem 212: 35-42.

Brito-Casillas Y, Figueirinhas P, Wiebe JC, López-Ríos L, Pérez-Barreto D, Melián C, Wägner AM (2014) ISO-based assessment of accuracy and precision of glucose meters in dogs. J Vet Intern Med 28: 1405-1413.

Clarke WL, Cox D, Gonder-Frederick LA, Carter W, Pohl SL (1987) Evaluating clinical accuracy of systems for self-monitoring of blood glucose. Diabetes Care 10: $622-628$

Cox DJ, Gonder-Frederick LA, Kovatchev BP, Julian DM, Clarke WL (1997) Understanding error grid analysis. Diabetes Care 20: 911-912.

Domori A, Sunahara A, Tateno M, Miyama TS, Setoguchi A, Endo Y (2014) The clinical utility of two human portable blood glucose meters in canine and feline practice. Vet Clin Pathol 43: 55-62.

Freckmann G, Schmid C, Baumstark A, Pleus S, Link M, Haug C (2012) System accuracy evaluation of 43 blood glucose monitoring systems for self-monitoring of blood glucose according to DIN EN ISO 15197. J Diabetes Sci Technol 6: 1060-1075.
Higgins PJ, Garlick RL, Bunn HF (1982) Glycosylated hemoglobin in human and animal red cells. Role of glucose permeability. Diabetes 31: 743-748.

ISO 15197 (2003) In Vitro Diagnostic Test Systems-Requirements for Blood-glucose Monitoring Systems for Self-testing in Managing Diabetes Mellitus. European Committee for Standardization (CEN): Brussels, http://www.iso.org/iso/catalogue_detail?csnumber $=26309$

ISO 15197 (2013) In Vitro Diagnostic Test Systems-Requirements for Blood-glucose Monitoring Systems for Self-testing in Managing Diabetes Mellitus. European Committee for Standardization (CEN): Brussels, http://www.iso.org/iso/catalogue_detail?csnumber $=54976$

Johnson BM, Fry MM, Flatland B, Kirk CA (2009) Comparison of a human portable blood glucose meter, veterinary portable blood glucose meter, and automated chemistry analyzer for measurement of blood glucose concentrations in dogs. $\mathbf{J}$ Am Vet Med Assoc 235: 1309-1313.

Khositseth A, Pharadornuwat O, Lertbunrian R, Anantasit N, Vaewpanich J (2015) Accuracy of bedside glucometry in critically ill children with peripheral hypoperfusion. J Clin Monit Comput 29: 145-152.

Mori A, Lee P, Yokoyama T, Oda H, Saeki K, Miki Y, Nozawa S, Azakami D, Momota Y, Makino Y, Matsubara T, Osaka M, Ishioka K, Arai T, Sako T (2011) Evaluation of artificial pancreas technology for continuous blood glucose monitoring in dogs. J Artif Organs 14: 133-139.

Magnani M, Dachg M, Bossw M, Fornaini G (1978) Comparative studies on red blood cell glucose phosphorylating activities of mammals. Comp Biochem Physiol B 60: 323-327.

Rendell M, Stephen PM, Paulsen R, Valentine JL, Rasbold K, Hestorff T, Eastberg S, Shint DC (1985) An interspecies comparison of normal levels of glycosylated hemoglobin and glycosylated albumin. Comp Biochem Physiol B 81: 819-822.

Sacks DB (2006) Carbohydrates. pp. 837-901. In: Tietz Textbook of Clinical Chemistry and Molecular Diagnostics. $4^{\text {th }}$ ed. (Burtis CA, Ashwood ER, Bruns DE, eds.), Elsevier Saunders, St. Louis, MO.

Wess G, Reusch C (2000a) Assessment of five portable blood glucose meters for use in cats. Am J Vet Res 61: $1587-1592$

Wess G, Reusch C (2000b) Capillary blood sampling from the ear of dogs and cats and use of portable meters to measure glucose concentration. J Small Anim Pract 41: $60-66$. 